\title{
The Simplest Neutrino Mass Matrix Revisited
}

\author{
P.F. Harrison* \\ University of Warwick \\ E-mail: p.f.harrisonewarwick.ac.uk \\ R. Krishnan \\ University of Warwick \\ E-mail:k.rama@warwick.ac.uk \\ W. G. Scott \\ Rutherford Appleton Laboratory \\ E-mail: w.g.scotterl.ac.uk
}

In 2004, two of us proposed a texture, the "Simplest" neutrino mass matrix, which predicted $\sin \theta_{13}=\sqrt{2 \Delta m_{\text {sol }}^{2} / 3 \Delta m_{\text {atm }}^{2}}$ and $\delta_{C P}= \pm 90^{\circ}$. Using today's measured values for neutrino masssquared differences, this prediction gives $\sin ^{2} 2 \theta_{13} \simeq 0.086_{-0.006}^{+0.003}$, in excellent agreement with a measured value of $\sin ^{2} 2 \theta_{13}=0.098 \pm 0.011$. Here we present a specific model based on $S_{4}$ symmetry leading to this successful texture in the context of the type-1 see-saw mechanism, assuming Majorana neutrinos. Similar predictions are obtained relating $\theta_{13}$ to the light neutrino masses, which are in accord with current experimental limits and testable at future experiments. Large $C P$ asymmetries remain a generic prediction of the texture.

36th International Conference on High Energy Physics

4-11 July 2012

Melbourne, Australia

${ }^{*}$ Speaker. 


\section{Introduction}

Leptonic mixing is characterised by two large mixing angles, $\theta_{12} \simeq 35^{\circ}$ and $\theta_{23} \simeq 45^{\circ}$, and one small one, $\theta_{13}$. For several years, the data on neutrino oscillations were compatible with $\theta_{13}=0$, and the data together were approximated by the tribimaximal (TBM) mixing scheme [1]. TBM has been used by many authors as a starting point for model building. The Daya Bay [2] and RENO [3] reactor neutrino experiments recently measured the value for the reactor mixing angle to be definitively non-zero, with a combined value of $\sin ^{2} 2 \theta_{13}=0.098 \pm 0.010$ (stat.) \pm 0.005 (syst.), a result given additional support by several other experiments $[4,5,6]$.

Anticipating possible deviations from TBM, two of us proposed some generalisations [7], in which the condition $\theta_{13}=0$ was relaxed variously. Eg. in a basis in which the charged lepton mass matrix (MM) is diagonal, a hermitian neutrino MM which has $\mu-\tau$ reflection symmetry [8] and democracy (each of its rows and columns sums to a common value) [9] leads to "tri $\chi$ maximal" mixing $(\mathrm{T} \chi \mathrm{M})[7]$ :

$$
\left|U_{e 2}\right|^{2}=\frac{1}{3}, \quad \sin ^{2} \theta_{23}=\frac{1}{2}, \quad \sin ^{2} \theta_{13}=\frac{1}{3}\left(1-\frac{1}{\sqrt{1+3 k^{2}}}\right) ; \quad \delta_{C P}= \pm 90^{\circ} .
$$

We remark that $\mathrm{T} \chi \mathrm{M}$ corresponds to maximal $C P$ violation, for any particular value of $\theta_{13}$.

If neutrinos were Majorana particles, the neutrino MM would be complex-symmetric. We therefore determined [10] the general complex-symmetric MM that generates $\mathrm{T} \chi \mathrm{M}$ :

$$
M_{S}=a\left(\begin{array}{ccc}
1 & -i k & i k \\
-i k & 1+i k & 0 \\
i k & 0 & 1-i k
\end{array}\right)+b\left(\begin{array}{lll}
0 & 1 & 1 \\
1 & 1 & 0 \\
1 & 0 & 1
\end{array}\right)+c\left(\begin{array}{lll}
1 & 0 & 0 \\
0 & 0 & 1 \\
0 & 1 & 0
\end{array}\right)
$$

with $k, a, b$, and $c$ real parameters. The only free mixing parameter in $\mathrm{T} \chi \mathrm{M}$ is the angle $\theta_{13}$ which is uniquely determined by the real parameter $k$ in the matrix $M_{S}$.

The T $\chi \mathrm{M}$ mixing form [7], $U_{T \chi M}$, defined by Eq. (1.1), diagonalises each of the three terms of the matrix $M_{S}$ of Eq. (1.2), independently. The first term of $M_{S}$ alone is in fact, sufficient to generate $\mathrm{T} \chi \mathrm{M}$, having three distinct eigenvalues. The second and the third terms of $M_{S}$ give two degenerate and three degenerate eigenvalues respectively. A symmetric matrix, $M_{S}$, can be diagonalised using the appropriate unitary matrix and its transpose to give real positive eigenvalues:

$$
U_{T \chi M}^{T} M_{S} U_{T \chi M}=\operatorname{Diag}\left(\left|a \sqrt{1+3 k^{2}}-b+c\right|,|a+2 b+c|,\left|-a \sqrt{1+3 k^{2}}-b+c\right|\right) .
$$

A special case of $\mathrm{T} \chi \mathrm{M}$, known as "Simplest" neutrino mixing and defined by $b=0$ (eg. in Eqs. (1.2) and (1.3)) was proposed in 2004 [11] (having been first introduced in [7]). Simplest neutrino mixing yields an exact and very simple relation between the reactor mixing angle (see Eq. (1.1)) and the eigenvalues, $e_{i}$ :

$$
\sin ^{2} \theta_{13}=\frac{2}{3} \frac{\left(e_{2}-e_{1}\right)}{\left(e_{3}-e_{1}\right)}
$$

In the original publications [7, 11], the Simplest texture was proposed for $M_{v}^{2}:=M_{v} M_{v}^{\dagger}$, in which case the eigenvalues are the neutrino masses-squared, resulting in the very successful prediction:

$$
\sin \theta_{13}=\sqrt{\frac{2}{3} \frac{\Delta m_{\mathrm{sol}}^{2}}{\Delta m_{\mathrm{atm}}^{2}}}
$$




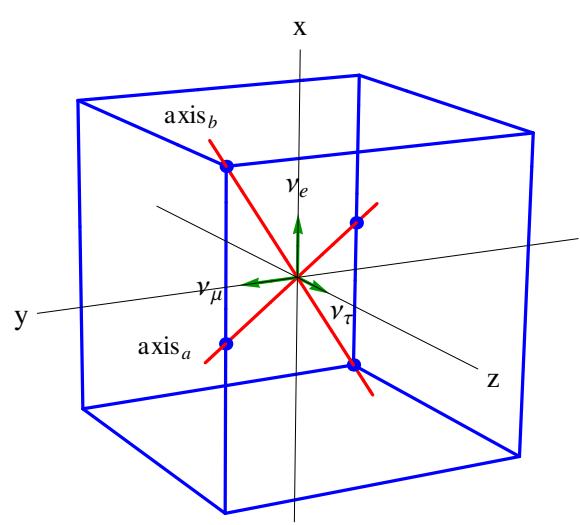

(a)

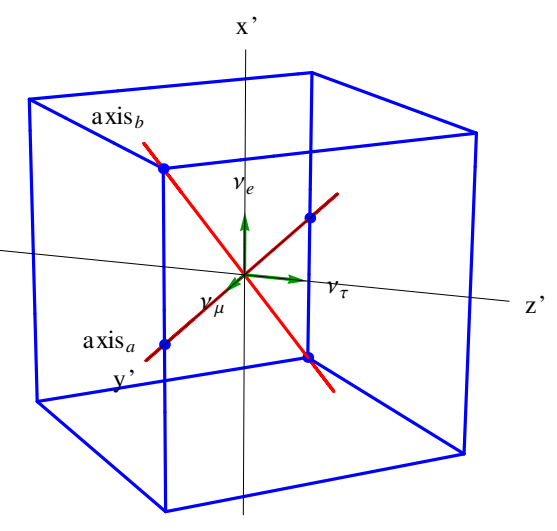

(b)

Figure 1: Octahedral symmetry in the $\mu-\tau$ rotated basis $\left(x^{\prime}, y^{\prime}, z^{\prime}\right)$.

i.e. $\sin ^{2} 2 \theta_{13}=0.086_{-0.006}^{+0.003}$, predicted in $2002 / 2004$ [7, 11], compared with $\sin ^{2} 2 \theta_{13}=0.098 \pm$ 0.011 , measured in 2012 [2,3].

In view of this very successful prediction, we decided to revisit Simplest mixing, proposing a new model for it [10], based on the group $S_{4}$. We make the following two changes with respect to the original ansatz: we adopt the Simplest form for the neutrino MM itself (rather than its hermitian square); a Majorana mass term is assumed, exploiting the type-I see-saw mechanism. Thus, we get a Majorana neutrino MM of the following Simplest complex-symmetric form:

$$
M_{v}(\text { Majorana })=a\left(\begin{array}{ccc}
1 & -i k & i k \\
-i k & 1+i k & 0 \\
i k & 0 & 1-i k
\end{array}\right)+c\left(\begin{array}{lll}
1 & 0 & 0 \\
0 & 0 & 1 \\
0 & 1 & 0
\end{array}\right) .
$$

We construct such a Majorana MM assuming symmetry under $S_{4}$, with the neutrino Dirac MM assumed to be proportional to the identity. We show that this model has a phenomenology compatible with experiment, and we use it to predict the masses of the light neutrinos.

\section{The group $S_{4}$ and the $\mu-\tau$ rotated basis}

$S_{4}$ is the symmetry group of the cube and the octahedron. It has the abstract presentation $\left\langle\boldsymbol{a}, \boldsymbol{b} \mid \boldsymbol{a}^{2}=\boldsymbol{b}^{3}=(\boldsymbol{a} \boldsymbol{b})^{4}=e\right\rangle$, where $\boldsymbol{a}, \boldsymbol{b}$ and $\boldsymbol{a} \boldsymbol{b}$ represent the orientation-preserving rotations of a cube through angles $\pi, \frac{2 \pi}{3}$ and $\frac{\pi}{2}$ respectively, as shown in Fig. 1 (axis ${ }_{a}$ and axis $_{b}$ correspond to the generators $\boldsymbol{a}$ and $\boldsymbol{b}$ respectively). The character table for the $S_{4}$ group can be found in [12].

We define a right-handed neutrino triplet $v_{R}=\left(v_{e}, v_{\mu}, v_{\tau}\right)$. Many authors work in a basis with the coordinate axes normal to the faces of the cube as shown in Fig. 1(a) and with the elements of $v_{R}$ defined parallel to these coordinate axes. Then the $v_{e}, v_{\mu}$ and $v_{\tau}$ are simply the invariant eigenstates (eigenstates with eigenvalue equal to +1 ) of the $\frac{\pi}{2}$ symmetry rotations of the cube. For our model of the "Simplest" texture, we define instead the $v_{\mu}$ and $v_{\tau}$ basis states rotated by an angle $\frac{\pi}{4}$ in the $y-z$ plane relative to the cube, as shown in Fig. 1(b). For covenience, we also rotate the $y$ and $z$ coordinate axes to align with the new $v_{\mu}$ and $v_{\tau}$ flavour basis states respectively, denoting 
the rotated coordinate system, $\left(x^{\prime}, y^{\prime}, z^{\prime}\right)$ as shown in Fig. 1(b). The state $v_{e}$ is unchanged and still corresponds to the $\frac{\pi}{2}$-rotation symmetry of the cube about the $x$-axis. However, $v_{\mu}$ and $v_{\tau}$ are no longer invariant eigenstates of $\frac{\pi}{2}$-rotations, but rather are invariant under $\pi$ rotations of the cube, as may be seen in Fig. 1(b). We call this new basis, the $\mu$ - $\tau$ rotated basis.

The three-dimensional representation of $S_{4}$ corresponding to the rotational symmetries of the cube is denoted by $\mathbf{3}^{\prime}$ here. Thus the neutrino triplet $\left(v_{e}, v_{\mu}, v_{\tau}\right)$ belongs to the $\mathbf{3}^{\prime}$ representation. A Majorana mass term contains two neutrino fields, and thus it is of interest to consider the tensor product decomposition of two $\mathbf{3}^{\prime}$ s. This decomposition is as follows:

$$
3^{\prime} \times 3^{\prime}=1+2+3+3^{\prime}
$$

where 1 is the trivial representation, and in the $\mu$ - $\tau$ rotated basis we have:

$$
\begin{aligned}
& \chi_{1}=\frac{1}{\sqrt{3}}\left(v_{e} \cdot v_{e}+v_{\mu} \cdot v_{\mu}+v_{\tau} \cdot v_{\tau}\right), \quad \chi_{2}=\left(\begin{array}{c}
-\sqrt{\frac{2}{3}} v_{e} \cdot v_{e}+\frac{1}{\sqrt{6}} v_{\mu} \cdot v_{\mu}+\frac{1}{\sqrt{6}} v_{\tau} \cdot v_{\tau} \\
\frac{1}{\sqrt{2}}\left(v_{\mu} \cdot v_{\tau}+v_{\tau} \cdot v_{\mu}\right)
\end{array}\right), \\
& \chi_{3}=\left(\begin{array}{c}
\frac{1}{\sqrt{2}}\left(v_{\mu} \cdot v_{\mu}-v_{\tau} \cdot v_{\tau}\right) \\
\frac{1}{\sqrt{2}}\left(v_{\tau} \cdot v_{e}+v_{e} \cdot v_{\tau}\right) \\
\frac{1}{\sqrt{2}}\left(v_{e} \cdot v_{\mu}+v_{\mu} \cdot v_{e}\right)
\end{array}\right), \quad \quad \chi_{3}^{\prime}=\left(\begin{array}{c}
\frac{1}{\sqrt{2}}\left(v_{\mu} \cdot v_{\tau}-v_{\tau} \cdot v_{\mu}\right) \\
\frac{1}{\sqrt{2}}\left(v_{\tau} \cdot v_{e}-v_{e} \cdot v_{\tau}\right) \\
\frac{1}{\sqrt{2}}\left(v_{e} \cdot v_{\mu}-v_{\mu} \cdot v_{e}\right)
\end{array}\right)=0,
\end{aligned}
$$

where the bi-linears $\chi_{1}, \chi_{2}, \chi_{3}, \chi_{3}^{\prime}$ transform as $1,2,3,3^{\prime}$ respectively. The product $v_{i} . v_{j}$ is the Lorentz-invariant product of the right-handed neutrino Weyl spinors.

We now assume three types of flavons, $\phi_{1}, \phi_{2}$ and $\phi_{3}$, which transform as 1, 2 and 3 respectively, allowing us to write an invariant mass term:

$$
\operatorname{Inv}=c_{1} \chi_{1} \phi_{1}+c_{2} \chi_{2}^{T} \phi_{2}+c_{3} \chi_{3}^{T} \phi_{3}
$$

where $c_{1}, c_{2}$ and $c_{3}$ are constants. Once the flavons acquire specific forms of vacuum expectation values (vevs), the required MM can be obtained from the invariant mass term given in Eq. (2.3). Suppose the flavons get vevs

$$
\left\langle\phi_{1}\right\rangle=1,\left\langle\phi_{2}\right\rangle=\left(-\frac{1}{2} \frac{\sqrt{3}}{2}\right),\left\langle\phi_{3}\right\rangle=(1,1,-1),
$$

the MM obtained will be

$$
M=c_{1} I+\frac{c_{2} \sqrt{3}}{2 \sqrt{2}}\left(\begin{array}{ccc}
\frac{2}{3} & 0 & 0 \\
0 & -\frac{1}{3} & 1 \\
0 & 1 & -\frac{1}{3}
\end{array}\right)+\frac{c_{3}}{\sqrt{2}}\left(\begin{array}{ccc}
0 & -1 & 1 \\
-1 & 1 & 0 \\
1 & 0 & -1
\end{array}\right)
$$

which is in the same form as Eq. (1.6), assuming $c_{1}$ and $c_{2}$ to be real and $c_{3}$ to be imaginary (where here the trace is removed from the second term and is absorbed into the first). The details of how to obtain the flavon vevs by minimisation of flavon potentials are given in the appendix to Ref. [10].

\section{The model}

The model is constructed in a Standard Model framework with the addition of heavy righthanded neutrinos. Light Majorana neutrinos are produced via a type-1 seesaw mechanism. The 
fermion and flavon content of the model is given in Table 1. The Standard Model Higgs field is assigned to the trivial representation of $S_{4}$.

\begin{tabular}{|c|c|c|c|c|c|c|c|c|c|c|c|c|}
\hline & $e_{R}$ & $\mu_{R}$ & $\tau_{R}$ & $L$ & $v_{R}$ & $\phi_{1}^{-}$ & $\phi_{1}$ & $\phi_{2}$ & $\phi_{3}$ & $\phi_{3 e}^{\prime}$ & $\phi_{3 \mu}^{\prime}$ & $\phi_{3 \tau}^{\prime}$ \\
\hline$S_{4}$ & $\mathbf{1}$ & $\mathbf{1}$ & $\mathbf{1}$ & $\mathbf{3}^{\prime}$ & $\mathbf{3}^{\prime}$ & $\mathbf{1}$ & $\mathbf{1}$ & $\mathbf{2}$ & $\mathbf{3}$ & $\mathbf{3}^{\prime}$ & $\mathbf{3}^{\prime}$ & $\mathbf{3}^{\prime}$ \\
\hline$C_{2 e}$ & -1 & 1 & 1 & 1 & 1 & 1 & 1 & 1 & 1 & -1 & 1 & 1 \\
\hline$C_{2 \mu}$ & 1 & -1 & 1 & 1 & 1 & 1 & 1 & 1 & 1 & 1 & -1 & 1 \\
\hline$C_{2 \tau}$ & 1 & 1 & -1 & 1 & 1 & 1 & 1 & 1 & 1 & 1 & 1 & -1 \\
\hline$C_{2 D}$ & -1 & -1 & -1 & -1 & 1 & -1 & 1 & 1 & 1 & 1 & 1 & 1 \\
\hline
\end{tabular}

Table 1: The flavour structure of the model. $L$ are the three left-handed lepton weak isospin doublets and $v_{R}$ are the three right-handed heavy neutrinos. The $\mathbf{3}^{\prime}$ representations are in the $\mu-\tau$ rotated basis and the 2 and the 3 representations are in the basis given by the tensor product expansion in the text.

The $C_{2}$ flavour symmetries $C_{2 e}, C_{2 \mu}$ and $C_{2 \tau}$ ensure that the flavons $\phi_{3 e}^{\prime}, \phi_{3 \mu}^{\prime}$ and $\phi_{3 \tau}^{\prime}$ couple only to $e_{R}, \mu_{R}$ and $\tau_{R}$ respectively. We thus obtain a diagonal MM for the charged leptons from a mass term of the form

$$
\left(y_{e} L^{\dagger} e_{R} \phi_{3 e}^{\prime}+y_{\mu} L^{\dagger} \mu_{R} \phi_{3 \mu}^{\prime}+y_{\tau} L^{\dagger} \tau_{R} \phi_{3 \tau}^{\prime}\right)(H / \Lambda)+H . C .
$$

where $H$ is the standard model Higgs, $\Lambda$ is the cut-off scale and the $y_{i}$ are coupling constants. After the weak gauge symmetry and the flavour symmetry are spontaneously broken, with the flavons $\phi_{3 e}^{\prime}, \phi_{3 \mu}^{\prime}$ and $\phi_{3 \tau}^{\prime}$, getting vevs of $(1,0,0)^{T},(0,1,0)^{T}$ and $(0,0,1)^{T}$ respectively, we get the required masses $m_{e}, m_{\mu}$ and $m_{\tau}$ for the charged leptons. We postulate a singlet flavon $\phi_{1}^{-}$and introduce another $C_{2}$ symmetry $C_{2 D}$ to allow only this singlet flavon to enter the Dirac mass term:

$$
y_{w} L^{\dagger} v_{R} \tilde{H}\left(\phi_{1}^{-} / \Lambda\right)+H . C .,
$$

where $\tilde{H}$ is the conjugate Higgs and $y_{w}$, a coupling. Thus the neutrino Dirac MM is proportional to the identity. The Majorana mass term containing the right-handed neutrinos is as follows:

$$
\left(y_{1} \chi_{1} \phi_{1}+y_{2} \chi_{2}^{T} \phi_{2}+i y_{3} \chi_{3}^{T} \phi_{3}\right) / \Lambda
$$

where the $\chi_{i}$ are given by the expressions in Eqs. (2.2) and the $y_{i}(i=1,2,3)$ are couplings leading to very heavy right-handed Majorana masses. This leads to a Majorana MM of the form given by Eq. (2.5) as explained in the previous section. The flavons $\phi_{1}, \phi_{2}$ and $\phi_{3}$ get the vevs given in Eqs. (2.4) and also with $\left\langle\phi_{1}^{-}\right\rangle=1$, we get the following $6 \times 6$ mass matrix, $M$, for the neutrinos:

$$
v_{\alpha}^{T} M v^{\alpha} ; \quad M=\left(\begin{array}{cc}
0 & M_{\text {Dir }} \\
M_{\text {Dir }} & M_{\text {Maj }}
\end{array}\right), \quad v=\left(\begin{array}{c}
v_{L}^{*} \\
v_{R}
\end{array}\right)
$$

with $v_{L}$ the left-handed neutrino triplet and where $M_{\text {Dir }}=\frac{y_{w}}{2 \Lambda} I$, and $M_{\text {Maj }}$ is of the required form given in Eqs. (1.6) and (2.5). Here, $M_{\text {Maj }}$ is at a very high mass scale and $M_{\text {Dir }}$ is of order the weak scale, so that the seesaw mechanism comes into play. The effective mass matrix, $M_{\mathrm{ss}}$, thus generated for the left-handed light neutrinos is in the form [13]

$$
M_{\mathrm{ss}}=-M_{\text {Dir }} M_{\mathrm{Maj}}^{-1} M_{\text {Dir }} .
$$

A matrix of the form $U_{T \chi M}$ diagonalises $M_{\mathrm{ss}}$ giving light neutrino masses proportional to $\left(1 / e_{1}\right)$, $\left(1 / e_{2}\right),\left(1 / e_{3}\right)$, where $e_{1}, e_{2}$ and $e_{3}$ are the eigenvalues of $M_{\text {Maj. }}$. 


\section{Fitting the model with experimental data}

The squared differences of the light neutrinos masses are known experimentally, $m_{2}^{2}-m_{1}^{2}=$ $75.9 \pm 2.1 \mathrm{meV}^{2},\left|m_{3}^{2}-m_{2}^{2}\right|=2430 \pm 130 \mathrm{meV}^{2}$. The eigenvalues of the Majorana mass matrix, $M_{\text {Maj }}$ from Eq. (1.6), are

$$
e_{1}=c+a \sqrt{1+3 k^{2}}, \quad e_{2}=c+a, \quad e_{3}=c-a \sqrt{1+3 k^{2}}
$$

( $a, c$ and $k$ are real in our model, given our earlier assumptions). Since the light neutrino masses are inversely proportional to these eigenvalues, we get

$$
\frac{m_{2}^{2}-m_{1}^{2}}{m_{3}^{2}-m_{2}^{2}}= \pm \frac{75.9 \pm 2.1}{2430 \pm 130}=\frac{\left(1 / e_{2}^{2}\right)-\left(1 / e_{1}^{2}\right)}{\left(1 / e_{3}^{2}\right)-\left(1 / e_{2}^{2}\right)}=-\frac{(r-s)^{2}(1-s)(1+2 r+s)}{(r+s)^{2}(1+s)(1+2 r-s)}
$$

where $r=c / a$ and $s=\sqrt{1+3 k^{2}}$. Using the observed reactor mixing angle, $\theta_{13}$, in Eq. (1.1), we can find the value of $k$ and use this in Eq. (4.2) to obtain the parameter $r$, and thus predict the values of the light neutrino masses. Eq. (4.2) is cubic in $r$, giving three real solutions for normal hierarchy and one for inverted hierarchy. One of the normal hierarchy solutions gives the wrong sign for the solar mass-squared difference, leaving three solutions compatible with data. Thus the predictions for the light neutrino masses fall into three sets, as shown in Fig. 2, where the best fit values are used. The predicted ranges for the mass of the neutrino eigenstate $v_{1}$, allowing for

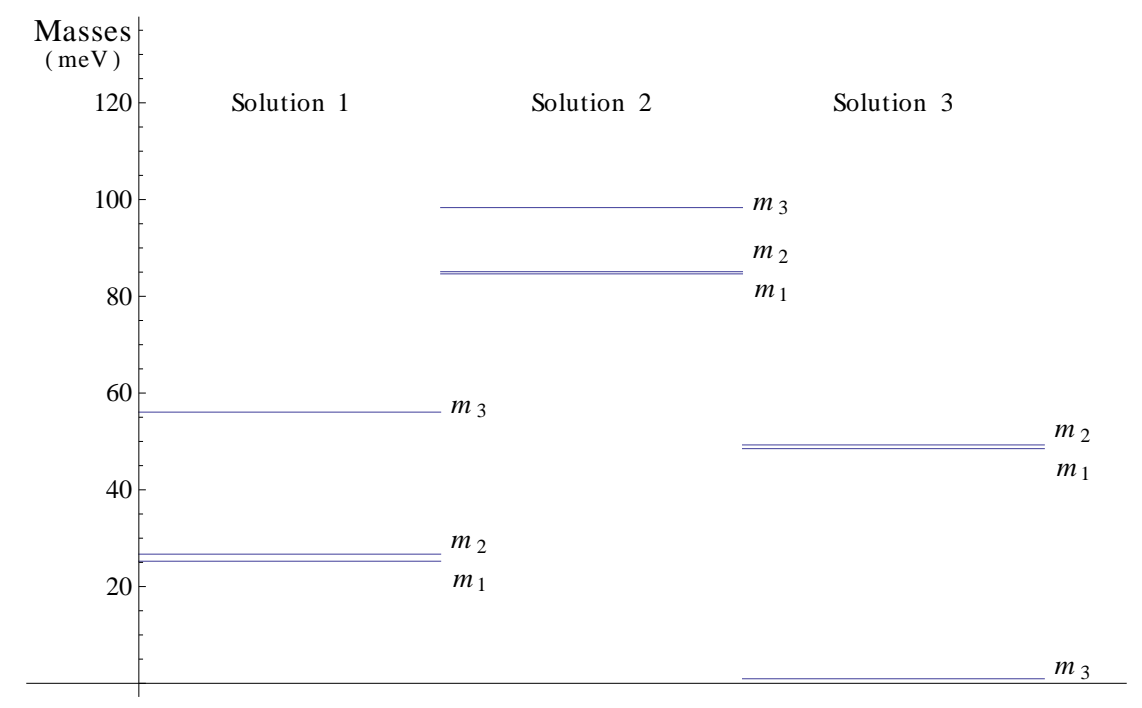

Figure 2: The predicted values of the neutrino masses corresponding to the best fit $\left(m_{2}^{2}-m_{1}^{2}=75.9 \mathrm{meV}^{2}\right.$, $\left.\left|m_{3}^{2}-m_{2}^{2}\right|=2430 \mathrm{meV}^{2}, \sin ^{2} 2 \theta_{13}=0.098\right)$. Case $1(r=0.4101)$ and case $2(r=14.452)$ are in normal hierarchy. Case 3 ( $r=-1.0405)$ is in inverted hierachy.

experimental uncertainties in the observed neutrino masss-squared differences are shown in Fig. 3. For solution 2 we do not consider a mass above $100 \mathrm{meV}$ in order to keep our prediction compatible with the cosmological upper limit of the masses of the neutrinos [14]. It should be emphasised that $\mathrm{T} \chi \mathrm{M}$, as obtained here implies maximal $C P$ violation $\left(\left|\delta_{C P}\right|=90^{\circ}\right)$. The positive and negative signs of the parameter $k$ correspond to the $C P$-violating phase $\delta_{C P}= \pm 90^{\circ}$. 


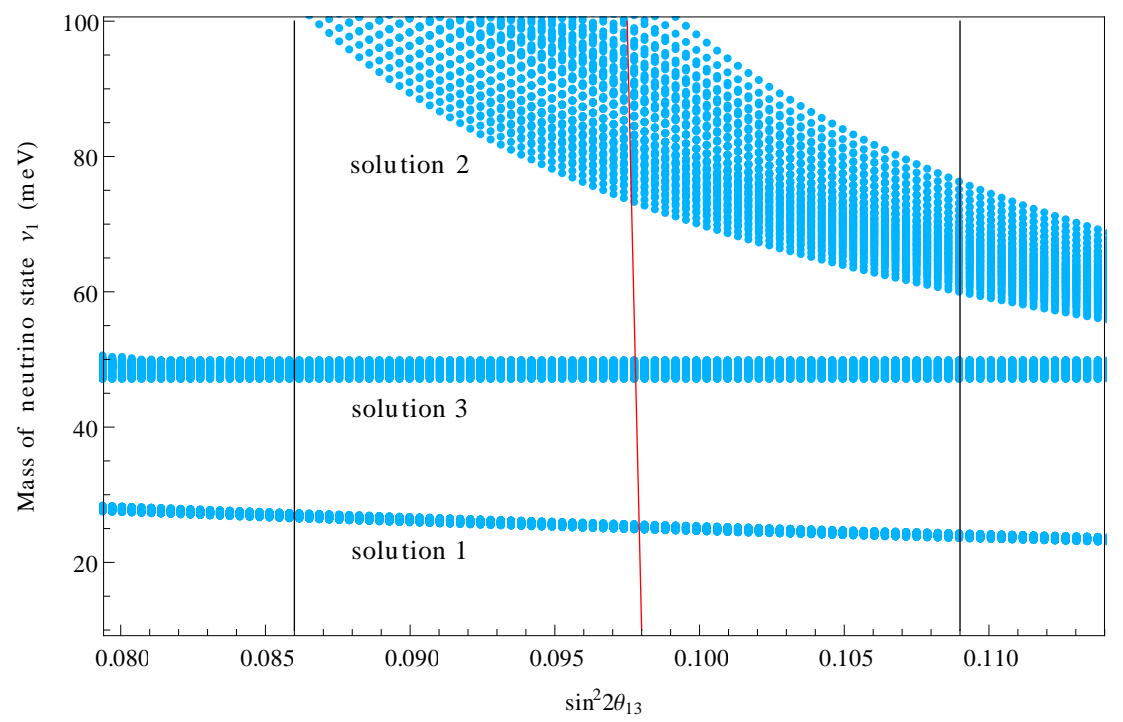

Figure 3: The predicted value of $m_{1}$ (the mass of the $v_{1}$ eigenstate) vs the measured value of $\sin ^{2} 2 \theta_{13}$. The finite thickness of the bands is due to the errors in the measurement of the neutrino mass-squared differences. The red and the black lines indicate the best fit value and the errors on $\sin ^{2} 2 \theta_{13}$ respectively.

This work was supported by the UK Science and Technology Facilities Council (STFC). PFH and RK acknowledge the hospitality of the Centre for Fundamental Physics (CfFP) at the Rutherford Appleton Laboratory. RK acknowledges support from CfFP and the University of Warwick.

\section{References}

[1] P. F. Harrison, D. H. Perkins, and W. G. Scott, Phys. Lett. B 530 (2002) 167, [hep-ph/ 0202074 ].

[2] F. P. An et al. (Daya Bay Collaboration), Phys. Rev. Lett. 108 (2012) 171803, [arXiv:1203.1669].

[3] J. K. Ahn et al. (RENO Collaboration), Phys. Rev. Lett. 108 (2012) 191802, [arXiv: 1204.0626 ].

[4] K. Abe et al. (T2K Collaboration), Phys. Rev. Lett. 107 (2011) 041801, [arXiv:1106.2822].

[5] P. Adamson et al. (MINOS Colln.), Phys. Rev. Lett. 107 (2011) 181802, [arXiv:1108. 0 015].

[6] Y. Abe et al. (DOUBLE-CHOOZ), Phys. Rev. Lett. 108 (2012) 131801, [arXiv:1112. 6353].

[7] P. F. Harrison and W. G. Scott, Phys. Lett. B 535 (2002) 163, [hep-ph/ 020320 9].

[8] P. F. Harrison and W. G. Scott, Phys. Lett. B 547 (2002) 219, [hep-ph / 0210197 ].

[9] P. F. Harrison and W. G. Scott, Phys. Lett. B 557 (2003) 76, [hep-ph/ 0302025 ].

[10] R. Krishnan, P.F. Harrison and W.G. Scott, [arXiv:1211.2000].

[11] P. F. Harrison and W. G. Scott, Phys. Lett. B 594 (2004) 324, [hep-ph/ 0403278 ].

[12] H. Ishimori et al, Prog. Theor. Phys. Suppl. 183 (2010) 1, [arXiv: 1003.3552$].$

[13] A. Y. Smirnov, Phys. Rev. D 48 (1993) 3264, [hep-ph/ 9304205 ].

[14] S. Riemer-Sorensen et al, (WiggleZ Colln.), Phys. Rev. D 85 (2012) 081101, [arXiv: 1112. 4940]. 